Computational and Mathematical Methods in Medicine

Vol. 10, No. 1, March 2009, 39-47

\title{
Computer-aided diagnosis of multiple sclerosis
}

\author{
R. Linder ${ }^{\mathrm{a} *}$, D. Mörschner ${ }^{\mathrm{b}}$, S.J. Pöppl ${ }^{\mathrm{a}}$ and A. Moser ${ }^{\mathrm{b}}$ \\ ${ }^{a}$ Institute of Medical Informatics, University of Lübeck, Lübeck, Germany; ${ }^{b}$ Department \\ of Neurology, University of Lübeck, Lübeck, Germany
}

(Received 18 December 2007; final version received 20 March 2008)

\begin{abstract}
The study aims to develop a computer-assisted decision support based on cerebrospinal fluid (CSF) and blood findings to improve their value and ease the diagnostic procedure of chronic inflammatory diseases (CIDs) of central nervous system (CNS). Data were collected from patients suffering from multiple sclerosis (MS, $n=73$ ), from another CID of the CNS $(n=22)$, or a psychiatric disease (control group, CTRL, $n=12$ ). Univariate and multivariate analyses were performed using multiple logistic regression and artificial neural networks. Differentiating between MS and CID, no parameter could be disclosed that could provide a meaningful decision support. However, multivariate analysis obtained a statistically significant classification (sensitivity = $84.9 \%$, specificity $=54.5 \%, p<0.001$ ). On the contrary, multivariate analysis based on the differentiation between MS vs. CTRL, gave good results (sensitivity $=95.9 \%$, specificity $=83.3 \%, p<0.001$ ). It became evident from standard laboratory findings that there is a significant potential for computer-aided decision support.
\end{abstract}

Keywords: multiple sclerosis; chronic inflammatory diseases of CNS; cerebrospinal fluid; artificial neural network; multiple logistic regression

\section{Introduction}

Compared to other neurological diseases, the diagnosis of multiple sclerosis (MS) is very complex. To cope with this complexity, McDonald et al. in 2001, declared a diagnostic criteria [23] model that is still valid after some recent revisions by Polman et al. [25]. The McDonald Criteria rely on a contemplation of clinical presentations (progression and relapses, and objective lesions) and further evidence from several objective findings (positive cerebrospinal fluid (CSF), positive visual evoked potentials and evident lesions in magnetic resonance imaging). McDonald Criteria constitute only two laboratory findings: the existence of oligoclonal IgG bands in CSF and an elevated IgG Index. If either of these conditions is satisfied, the CSF is rated as positive even though both laboratory findings are only indicative and are by no means critical. However there are no existing paraclinical features with sufficient sensitivity or specificity. Each finding given by McDonald Criteria might also occur in several other inflammatory diseases of the central nervous system (CNS). In this regard, the German Society for Neurology (DGN) proposed the likely presentation of vasculitides (an inflammation of blood vessels) with cerebral manifestation, neuro-sarcoidosis (a disease with small inflammatory nodules in nervous tissue of unknown aetiology) and borreliosis, also known as lyme disease (a bacterial

*Corresponding author. Email: linder@imi.uni-luebeck.de 
infection with the tick as vector). Furthermore, cerebral human immunodeficiency virus (HIV) manifestation and neurosyphilis constitute additional options [10,11,27]. In clinical practice of MS, the broad spectrum of chronic inflammatory diseases (CIDs) of the CNS leads to several further examinations, which account for additional costs due to the extended diagnostic procedure.

Consequently, a computer-assisted decision (CAD) support may be desirable that suffices with only a few findings achieving a high accuracy in the identification of MS patients. The present study especially focuses on CAD support of standard laboratory findings, i.e. laboratory blood findings and CSF findings. Findings obtained from MS patients were compared to the findings from psychiatric patients considered not to have an organic involvement, as well as to patients suffering from other inflammatory diseases of the CNS. The study is an attempt to characterize the prevalent profiles of findings in CSF and blood analyses in each disease and to determine if significant differences could be detected that may facilitate the diagnostic procedure for MS.

\section{Patients and methods}

\section{Data acquisition}

Data were collected in a hospital with maximum medical care, a clinic responsible for half of the population of Schleswig-Holstein (the very northern federal state in Germany). The results of all CSF punctures conducted during 2001 and 2002 were entered into a database, amounting to a total of 2879 punctures. Thousand three hundred and ninety-six of these punctures were applied to in-patients while the remaining were subjected to external patients of neighbouring clinics or general practitioners who had sent material for analysis. The majority of these patients suffered from a disease irrelevant to this study; just 95 patients suffered from either MS, or another CID of the CNS and were therefore relevant for the present study. For further analysis, patient records were obtained for in-patients and it was verified whether patients had been diagnosed with MS or another CID of the CNS. As CSF punctures from a healthy control group (CTRL) were not available, so an alternative control was needed. Therefore, patients with first manifestation of a psychiatric disease without organic involvement were included as CTRL. All of the MS patients fulfilled the McDonald Criteria.

Since each puncture was accompanied by blood findings, a total number of 33 parameters was analysed (Table 1). The Methods used in laboratory examinations reflect established current state-of-art methods, e.g. for the detection of oligclonal bands isolectric focussing has been carried out [5].

\section{Data analysis}

As pre-processing of data, categorical parameters, e.g. the macroscopic aspect before centrifugation, were re-coded into corresponding dummy variables using the 1-of-C coding scheme where the number of outputs is the same as the number of classes [4]. Re-coding the 34 parameters led to a total number of 43 variables. Missing data (1.1\%) were replaced by the mode for each variable as recommended by Lee et al. [15]. In contrast to the method proposed by Lee et al., other modes were then calculated not only for each variable but also for each class (MS, CID and CTRL).

The data analysis focussed on two two-class classification problems: MS vs. CID and MS vs. CTRL. For each of the two problems, three kinds of analyses were performed each with increasing complexity: 
Table 1. Standard laboratory findings by CSF puncture and the corresponding blood examination.

\begin{tabular}{|c|c|c|}
\hline CSF findings & Blood findings & $\begin{array}{c}\text { Quotients } \\
\text { (blood and CSF) }\end{array}$ \\
\hline \multicolumn{3}{|l|}{ Metabolic analyses } \\
\hline CSF glucose & Serum glucose & \\
\hline CSF lactate & Serum lactate & \\
\hline \multicolumn{3}{|l|}{ Protein analyses } \\
\hline Total CSF protein & Total serum protein & \\
\hline Total CSF IgA & Total serum IgA & IgA index \\
\hline Total CSF IgM & Total serum IgM & IgM index \\
\hline Total CSF IgG & Total serum IgG & IgG index \\
\hline Total CSF albumin & Total serum albumin & IgA CSF/serum \\
\hline \multirow[t]{6}{*}{ Oligoclonal bands ${ }^{\mathrm{a}}$} & Oligoclonal bands ${ }^{\mathrm{a}}$ & IgG CSF/serum \\
\hline & Albumin ${ }^{\mathrm{b}}$ & \\
\hline & $\alpha-1$-Globulin ${ }^{\mathrm{b}}$ & \\
\hline & $\alpha-2$-Globulin ${ }^{\mathrm{b}}$ & \\
\hline & $\beta$-Globulin ${ }^{\mathrm{b}}$ & \\
\hline & $\gamma$-Globulin ${ }^{\mathrm{b}}$ & \\
\hline
\end{tabular}

Cellular analyses

Macroscopic aspect before centrifugation

Lucent, cloudy, sanguineous

Macroscopic aspect after centrifugation

Lucent, cloudy, haemolytic

Erythrocytes

Leukocytes

Prevalence of plasma cells

Lymphomonocytic pleocytosis

Physiological histology:

No pleocytosis, normal cell relation, count

and morphology

${ }^{\mathrm{a}}$ Oligoclonal bands were detected by isoelectric focusing.

${ }^{\mathrm{b}}$ Electrophoretic fraction in serum.

(1) Univariate analysis examining the distinguishing power of each single parameter.

(2) Multiple logistic regression (MLR) investigating two sets of parameters, MLR2 and MLR5. The first set MLR2 comprised of the two parameters: IgG Index and oligoclonal bands mentioned in the McDonald Criteria. In addition, MLR5 consisted of another three parameters: total CSF protein, typical histological aspect with pleocytosis (increased number) of lymphocytes and mononuclear cells and cells per $\mu \mathrm{l}$ in CSF.

(3) Artificial neural network (ANN) analysis performing a feature selection starting from all 43 parameters available.

Although a three-way classification might seem possible, we considered this to be less fitting to the concept of our study: the main question was if classification allows differentiation of MS vs. CID. As this in clinical everyday life constitutes the difficulty, because of the likewise presentation of the MS and diseases summarized as CID. The differentiation between MS vs. healthy individuals (CTRL) in this respect seems less important. Another aspect is that a three-way classification would have brought up the necessity to include as well these patients who do not yet fulfil McDonald Criteria, a group of which we recently still lack knowledge. 


\section{Univariate analysis}

The univariate analysis was restricted to those parameters already known to play a role in the diagnosis of MS, which included: IgG Index (MS: >0.7), oligoclonal bands (MS: present), lymphomonocytic pleocytosis (MS: yes), and cells per $\mu 1$ in CSF (MS: in between 4 and 35). The thresholds applied are widely accepted $[1,8,12,14,20,22,24,30]$.

\section{Multiple logistic regression}

MLR models are the classical approach to predict continuous values. MLR was first described in 1894 by Galton [7], and since then it has become an inherent part of data analysis in contemporary life sciences. MLR is the method of choice when estimation is needed of dichotomy dependent variables that are based on multiple independent variables. In the medical field, this could be the assignment to one of the two diseases based on patients' characteristics. MLR is provided by a wide range of statistics software and can easily be applied by non-experts. Results of a regression formula can easily be interpreted. The following equation:

$$
y=\frac{1}{1+\mathrm{e}^{-\left(b_{1} \cdot x_{1}+b_{2} \cdot x_{2}+\cdots+b_{n} \cdot x_{n}+\text { offset }\right)}} ;
$$

is used to predict the dependent variable $y$, whereby the regression coefficients $\left(b_{1}-b_{n}\right)$ reflect the influence of the independent variables $x_{1}-x_{n}$. If $y$ exceeds a pre-defined threshold, e.g. 0.5, a patient will be assigned to one class or otherwise to the other class. It is assumed that both the independent variables do not interact with each other and that there are exclusively linear relationships between the $y$ - and $x$-variables.

MLR was calculated by the software SPSS V.13 (SPSS Inc., Chicago, USA), exclusively on default settings.

\section{Artificial neural network}

ANN technology is increasingly applied in data classification in the biomedical sciences $[6,21]$. The functionality of an ANN is derived from the brain as its biological archetype. Applying simple mechanisms of the neuronal processing, as far as the brain has been understood, one can train a network of artificial neurons to precisely classify the dependent variable [18]. A weight is assigned to each connection between two neurons. Based on nonlinear mathematical equations, an ANN iteratively establishes relationships between input and output variables by gradually adapting the weight factors assigned to inter-layer connections between artificial neurons. The system starts with random weights. Apparently optimal interconnection weights are iteratively 'learned' and stored ('experience') [9], and subsequently, the ANN should be organized to compute data based on fixed weight factors. Due to the mutual interconnection of its neurons and its sigmoidal activation functions, the ANN is capable of detecting interdependencies and nonlinearities. The so-called 'general function approximation theorem' suggests that a three-layered ANN with appropriate weights could approximate any arbitrary nonlinear function [13].

A feature selection was performed by sequential backward feature selection using the 'neural net clamping technique' published by Wang et al. [29]. For feature selection and training, the ANN prototypical software named Approximation and Classification of Medical Data (ACMD) [19] was applied. ANN and MLR were validated by the 'leaveone-out' method, i.e. training was established by using $n-1$ of all cases and the missing 
case was used for validation. By performing $n$ rounds of allocation, every case is used once for the purpose of validation.

\section{Results}

Most of the 1396 punctures were taken from patients suspicious for suffering from a CID of the CNS (26\%). Viral or bacterial infections of the meninges and the brain (18\%) were also frequent indications, whereas others belonged to more than 30 different indications with a broad spectrum of neurological diseases. (See Table 2 for the characteristics of the 107 patients included in the present study).

The results of the univariate and multivariate analyses are shown in Table 3 (MS vs. CID) and Table 4 (MS vs. CTRL). While sensitivity constitutes the percentage of correctly classified MS patients, specificity is the percentage of correctly classified non MS patients. Accuracy denotes the percentage of the total amount of correctly classified patients.

Distinguishing MS $(n=73)$ from CID $(n=22)$, IgG Index and oligoclonal bands as definition criteria of McDonald for MS demonstrate a sensitivity of 56.3\% (IgG Index) and

Table 2. Characteristics of patients in the present study.

\begin{tabular}{llccc}
\hline Group & Diagnosis & Patients & Sex M/F & Age (median) \\
\hline MS (n=73) & Primary progressive & 7 & $5 / 2$ & 50 \\
& Relapsing-remitting (full) & 27 & $6 / 21$ & 37 \\
& Relapsing-remitting (partial) & 29 & $9 / 20$ & 39 \\
& Secondary progressive & 10 & $7 / 3$ & 50 \\
CID $(n=22)$ & Neuroborreliosis stage III & 3 & $1 / 2$ & 60 \\
& Vasculitides of CNS & 9 & $3 / 6$ & 47 \\
& Neurosyphilis & 4 & $3 / 1$ & 55 \\
& Neurosarcoidosis & 2 & $1 / 1$ & 52 \\
CTRL $(n=12)$ & Cerebral manifestation of HIV & 4 & $1 / 3$ & 45 \\
& Patients with first manifestation & 12 & $6 / 6$ & 38 \\
& of psychiatric disease without & & & \\
& organic involvement & & \\
\hline
\end{tabular}

The groups summarize patients suffering from multiple sclerosis (MS), from another chronic inflammatory disease of the central nervous system (CID), or a psychiatric disease (CTRL).

Table 3. MS vs. CID of the CNS.

\begin{tabular}{lccccr}
\hline Approach & $\begin{array}{c}\text { Number of } \\
\text { parameters }\end{array}$ & $\begin{array}{c}\text { Sensitivity } \\
(\%)\end{array}$ & $\begin{array}{c}\text { Specificity } \\
(\%)\end{array}$ & $\begin{array}{c}\text { Accuracy } \\
(\%)\end{array}$ & $\chi^{2}(p)$ \\
\hline IgG index & 1 & 56.3 & 45.5 & 53.8 & 0.915 \\
Oligoclonal bands & 1 & 80.8 & 36.4 & 70.5 & 0.174 \\
Lymphomonocytic & 1 & 46.6 & 63.6 & 50.5 & 0.553 \\
pleocytosis & 1 & 52.1 & 59.1 & 53.7 & 0.501 \\
CSF cells per $\mu \mathrm{l}$ & 2 & 94.5 & 22.7 & 77.9 & 0.046 \\
MLR2 & 5 & 94.5 & 22.7 & 77.9 & 0.046 \\
MLR5 & 10 & 84.9 & 54.5 & 77.9 & $<0.001$ \\
ANN & & & & & \\
\hline
\end{tabular}

Sensitivity constitutes the percentage of correctly classified MS patients, specificity is the percentage of correctly classified CID patients and accuracy denotes the percentage of the total amount of correctly classified patients. MLR2 and MLR5 depict parameter sets investigated by MLR and ANN refers to the parameters used to train the ANN. The $\chi^{2}$ statistic was applied to a $2 \times 2$ contingency table comprising the prediction (MS yes or no) and the true diagnosis (MS yes or no). 
Table 4. MS vs. CTRL.

\begin{tabular}{lccccr}
\hline Approach & $\begin{array}{c}\text { Number of } \\
\text { parameters }\end{array}$ & $\begin{array}{c}\text { Sensitivity } \\
(\%)\end{array}$ & $\begin{array}{c}\text { Specificity } \\
(\%)\end{array}$ & $\begin{array}{c}\text { Accuracy } \\
(\%)\end{array}$ & $\chi^{2}(p)$ \\
\hline IgG index & 1 & 56.3 & 100 & 62.6 & 0.001 \\
Oligoclonal bands & 1 & 80.8 & 100 & 83.5 & $<0.001$ \\
Lymphomonocytic & 1 & 46.6 & 100 & 54.1 & 0.007 \\
pleocytosis & 1 & 52.1 & 100 & 58.8 & 0.003 \\
CSF cells per $\mu \mathrm{l}$ & 2 & 94.5 & 75.0 & 91.8 & $<0.001$ \\
MLR2 & 5 & 95.9 & 83.3 & 94.1 & $<0.001$ \\
MLR5 & 19 & 95.9 & 66.7 & 91.8 & $<0.001$ \\
ANN & & & &
\end{tabular}

Sensitivity constitutes the percentage of correctly classified MS patients, specificity is the percentage of correctly classified CTRL patients and accuracy denotes the percentage of the total amount of correctly classified patients. MLR2 and MLR5 depict parameter sets investigated by MLR and ANN refers to the parameters used to train the ANN. The $\chi^{2}$ statistic was applied to a $2 \times 2$ contingency table comprising the prediction (MS yes or no) and the true diagnosis (MS yes or no).

$80.8 \%$ (oligoclonal bands), while a specificity of 45.5 and $36.4 \%$, respectively, which accounts for an accuracy of 53.8 and $70.5 \%$. The single parameters, lymphomonocytic pleocytosis and cells per $\mu$ l in CSF, show less sensitivity (46.6 and $52.1 \%$ ) but better specificity (63.6 and 59.1\%). The MLR approaches detect sensitivity very accurately employing the parameter sets MLR2 and MLR5 with 94.5\%; however, they lack specificity $(22.7 \%)$. The ANN performs much better compared to all other approaches including the two parameters of McDonald Criteria, with a sensitivity of $84.9 \%$, a specificity of $54.5 \%$ and an accuracy of $77.9 \%$.

Distinguishing MS $(n=73)$ from CTRL $(n=12)$, the accuracy is higher than for MS vs. CID in all approaches, i.e. the classification of MS vs. CTRL is less complex. Again, the multivariate approaches MLR2, MLR5 and ANN, obtained a higher accuracy than each of the single parameters (MLR2 and ANN: 91.8\%, MLR5: 94.1\%), with the most accurate single parameter being the oligoclonal bands with $83.5 \%$ accuracy.

To give an impression about the saliency of the parameters used in the multivariate approaches, MLR2, MLR5 and ANN were specified in all patients suffering from MS and CID. As a result, the regression coefficient for the $\mathrm{IgG}$ index was -0.312 , the coefficient for the oligoclonal bands was 1.001, and the offset was 0.752 . The regression coefficient for the IgG Index is negative because the CID patients present higher IgG indexes than MS patients. The coefficients can be directly processed by Equation (1). If $y$ exceeds 0.5 , a patient will be assigned to MS, otherwise to CID. Specifying an ANN with the same patients, the feature selection reveals the following nine parameters to be relevant: sex, CSF glucose, serum lactate, lymphomonocytic pleocytosis, physiological histology (no pleocytosis, normal cell relation, count and morphology), clear macroscopic aspect before centrifugation, albumin (electrophoretic fraction in serum and total serum concentration), and $\beta$-Globulin (serum).

Specifying the MLR with all patients suffering from MS and CTRL using the approach MLR5, the regression coefficients are as follows: $x_{1}=\operatorname{IgG}$ Index, $b_{1}=70.361, x_{2}=$ oligoclonal bands, $b_{2}=12.923, x_{3}=$ total CSF protein, $b_{3}=0.042, x_{4}=$ typical lymphomonocytic pleocytosis, $b_{4}=-3.308, x_{5}=$ total cells per $\mu \mathrm{l}$ in CSF, $b_{5}=0.617$, and an offset of -39.088 . Again, a functional value $y$ greater than 0.5 was assigned to MS. The regression coefficient of lymphomonocytic pleocytosis is negative because the majority of the MS patients (39 out of 73) did not show a lymphomonocytic pleocytosis. The analogue ANN comprised 19 input neurons for the same number of parameters. 


\section{Discussion}

In a northern German hospital providing maximum medical care, we found a group of 73 MS patients in the period from 2001 to 2002. Considering the course of the disease, the progressive forms (comprising 17 patients) showed predominantly men (12) and a minority of women $(5)$ (male-female ratio $=2.4: 1)$ with a median age of 50 years. The relapsing forms presented 56 patients with 41 women and 15 men (male-female ratio $=1: 2.7$ ) with a median age of 38.5 years. The overall male-female ratio was close to $1: 2$, in line with the large epidemiological study by Beer and Kesselring including 1016 individuals [3]. Male domination in progressive courses is not to be found in other studies. Even if this result is considered with caution due to the small sample size $(n=17)$, it is in agreement with Beer and Kesselring who found in their epidemiological study that the progressive forms are less female dominated (progressive courses: 1:1.3, relapsing courses: 1:1.9).

Contemplating the diagnostic value of the two features IgG Index and oligoclonal CSF bands given by McDonald Criteria [23], focussing on our given field of CIDs of CNS, we do notice a lack of discriminatory power. While sensitivity is $56.3 \%$ for the $\operatorname{IgG}$ Index and $80.8 \%$ for oligoclonal bands the specificity is merely 45.5 and $36.4 \%$, respectively. These results are not in line with the body of literature $[5,26,28]$, which usually refers to a greater spectrum of neurological diseases and does not (in contrast to the present study) focus on CIDs of the CNS. For this reason, the diagnostic value of IgG Index and oligoclonal bands seems to be overestimated. This is even more so, because usually the literature does not include the high number of patients, who do not fulfil McDonald Criteria for the diagnosis of MS. A certain number of these patients will develop MS in the future, while others do not. Therefore, when calculating sensitivity and specificity this group has to be integrated and highly reduces the diagnostic value of IgG Index and oligoclonal bands. In our clinic this group was the most numerous of all, with 96 members, compared with 73 individuals suffering from MS. Because of the still unsolved problem in diagnosis of MS, whether this group should be considered healthy or not, we did not integrate it in our study. However, for a realistic estimation of sensitivity and specificity, consideration of these patients should be obligatory.

Considering IgG Index we found an elevation largely for CID than for MS, e.g. cerebral manifestation of HIV-virus and neurosyphilis regularly presented perspicuously elevated $\operatorname{IgG}$ indexes higher than 0.7 .

The poor distinguishing power of laboratory features forced the authors to improve the authenticity of these by installing a multivariate analysis in diagnostic procedure of MS. To our knowledge such an approach was undertaken for the first time.

The present study is a proof-of-principle of whether the diagnostic procedure of MS can be facilitated by a low cost computer-aided diagnosis based only on a few standard laboratory parameters. Starting from CSF findings and blood examinations, it could be demonstrated that MS can be differentiated from other CIDs of CNS in a statistically significant manner. The same holds for the differential diagnosis of MS vs. CTRL consisting of patients with first manifestation of psychiatric disease without organic involvement.

Regarding MS vs. CID, the ANN approach was classified as the most accurate with a sensitivity $=84.9 \%$ and a specificity $=54.4 \%$. The advantage of ANNs is that they reveal nonlinear relationships and have the ability to analyse the interaction between many variables at different levels. For example, there is evidence that the superiority of ANNs is influenced by their ability to adjust the importance of certain parameters depending on the presence or absence of other variables [2]. The grouping of several findings is more profitable than any univariate analysis. 
Regarding MS vs. CTRL, the highest accuracy was obtained by the MLR approach using the parameter set MLR5 (sensitivity $=95.9 \%$, specificity $=83.3 \%$ ). This is evidently more accurate than results achieved by the use of a single parameter in the present study. Compared to MLR the ANN approach did slightly worse, which could be due to a non-optimal feature selection. (For a more general comparison between MLR and ANN please refer elsewhere) [16,17]. Further aspects not yet considered are data cleaning, missing data, and the effect of incomplete data on MLR and ANN. Of course there are other methodical approaches that work out different aspects, such as a classification tree seeking interaction effects, or support vector machines that provide another way to explore nonlinearities. However, this study is a proof of principle. Such methods should be varied in future studies with more data, comparing their profiles of performance.

Due to the relatively small sample size (CID: $n=22$; CTRL: $n=12$ ), the results should be considered with caution. On the contrary, the unexpectedly robust leave-oneout-validated results are even more very encouraging. In future, further investigations will be undertaken including a larger sample size, a separate consideration of the very inhomogeneous group of CIDs of the CNS, and an expansion to further examinations such as visual evoked potentials or magnetic resonance imaging. Even if the present results prove to be suitable for routine clinical use, a future CAD support seems feasible.

\section{References}

[1] M. Andersson, J. Alvarez-Cermeño, G. Bernardi, I. Cogato, P. Fredman, J. Frederiksen, S. Fredrikson, P. Gallo, L.M. Grimaldi, and M. Grønning, Cerebrospinal fluid in the diagnosis of multiple sclerosis: A consensus report, J. Neurol. Neurosurg. Psychiatry 57 (1994), pp. 897-902.

[2] W.G. Baxt, Application of artificial neural networks to clinical medicine, Lancet 346 (1995), pp. $1135-1138$.

[3] S. Beer and J. Kesselring, High prevalence of multiple sclerosis in Switzerland, Neuroepidemiology 13 (1994), pp. 14-18.

[4] C.M. Bishop, Neural Networks for Pattern Recognition, Clarendon Press, Oxford, 1995.

[5] A. Bourahoui, J. De Seze, R. Guttierez, B. Onraed, B. Hennache, D. Ferriby, T. Stojkovic, and P. Vermersch, CSF isoelectrofocusing in a large cohort of MS and other neurological diseases, Eur. J. Neurol. 11 (2004), pp. 525-529.

[6] R. Dybowski, Clinical Applications of Artificial Neural Networks, University Press, Cambridge, 2001.

[7] F. Galton, Natural Inheritance, 5th ed., Macmillan and Company, New York, 1894.

[8] K. Ganrot and C.B. Laurell, Measurement of IgG and albumin content of cerebrospinal fluid, and its interpretation, Clin. Chem. 20 (1974), pp. 571-573.

[9] C.C. Geddes, J.G. Fox, M.E.M. Allison, J.M. Boulton-Jones, and K. Simpson, An artificial neural network can select patients at high risk of developing progressive IgA nephropathy more accurately than experienced nephrologists, Nephrol. Dial. Transplant. 13 (1998), pp. 67-71.

[10] S.J. Greenberg, Human retroviruses and demyelinating diseases, Neurol. Clin. 13 (1995), pp. $75-97$.

[11] E.A. Hische, J.A. Tutuarima, E.C. Wolters, L. van Trotsenburg, R.V. van Eyk, JD Bos, A. Albert, and H.J. van der Helm, Cerebrospinal fluid $\operatorname{Ig} G$ and IgM indexes as indicators of active neurosyphilis, Clin. Chem. 34 (1988), pp. 665-667.

[12] E.A. Hische and H.J. van der Helm, Rate of synthesis of IgG within the blood-brain barrier and the IgG index compared in the diagnosis of multiple sclerosis, Clin. Chem. 33 (1987), pp. 113-114.

[13] K. Hornik, M. Stinchcombe, and H. White, Multilayer feedforward networks are universal approximators, Neural Netw. 2 (1989), pp. 359-366.

[14] J. Kesselring, Multiple Sklerose, Kohlhammer, Stuttgart, 2005.

[15] I.N. Lee, S.C. Liao, and M. Embrechts, Data mining techniques applied to medical information, Med. Inform. Internet Med. 25 (2000), pp. 81-102.

[16] R. Linder, J. Geier, and M. Kölliker, Artificial neural networks, classification trees and regression: Which method for which customer?, Database Mark. Customer Strateg. Manag. 11 (2004), pp. 344-356.

[17] R. Linder, I. König, C. Weimar, H.C. Diener, S.J. Pöppl, and A. Ziegler, Two models for outcome prediction: A comparison of logistic regression and neural networks, Methods Inf. Med. 45 (2006), pp. 536-540.

[18] R. Linder, E.I. Mohamed, A. De Lorenzo, and S.J. Pöppl, About the capabilities of artificial neural networks in body-composition research, Acta. Diabetol. 1(40 Suppl) (2003), pp. 9-14.

[19] R. Linder and S.J. Pöppl, ACMD: A practical tool for automatic neural net based learning, Lect. Notes Comp. Sci. 2199 (2001), pp. 168-173. 
[20] H. Link and G. Tibbling, Principles of albumin and IgG analyses in neurological disorders. III. Evaluation of IgG synthesis within the central nervous system in multiple sclerosis, Scand. J. Clin. Lab. Invest. 37 (1977), pp. 397-401.

[21] H. Malmgren, M. Borga, and L. Niklasson, Artificial Neural Networks in Medicine and Biology, Proceedings of the Annimab-1 Conference 2000, Springer, Telos, 2000.

[22] R. Mandler, H. Goren, and R. Valenzuela, Value of central nervous system IgG daily synthesis determination in the diagnosis of multiple sclerosis, Neurology 32 (1982), pp. 296-298.

[23] W.I. McDonald, A. Compston, G. Edan, D. Goodkin, H.P. Hartung, F.D. Lublin, H.F. McFarland, D.W. Paty, C.H. Polman, S.C. Reingold, M. Sandberg-Wollheim, W. Sibley, A. Thompson, S. van den Noort, B.Y Weinshenker, and J.S. Wolinsky, Recommended diagnostic criteria for multiple sclerosis: Guidelines from the international panel on the diagnosis of multiple sclerosis, Ann. Neurol. 50 (2001), pp. 121-127.

[24] J. Noseworthy, D. Paty, T. Wonnacott, T. Feasby, and G. Ebers, Multiple sclerosis after age 50, Neurology 33 (1983), pp. $1537-1544$

[25] C.H. Polman, S.C. Reingold, G. Edan, M. Filippi, H.P. Hartung, L. Kappos, F.D. Lublin, L.M. Metz, H.F McFarland, P.W. O'Connor, M. Sandberg-Wollheim, A.J. Thompson, B.J. Weinshenker, and J.S. Wolinsky, Diagnostic criteria for multiple sclerosis: 2005 revisions to the McDonald Criteria, Ann. Neurol. 58 (2005), pp. $840-846$

[26] I. Siddiqui, S. Aleem, N. Kayani, and S. Baig, CSF oligoclonal bands in multiple sclerosis, J. Pak. Med. Assoc. 52 (2002), pp. 351-353.

[27] M. Timmermans and J. Carr, Neurosyphilis in the modern era, J. Neurol. Neurosurg. Psychiatry 75 (2004), pp. $1727-1730$.

[28] L.M. Villar, J. Masjuan, M.C. Sadaba, P. Gonzalez-Porque, J. Plaza, A. Bootello, and J.C. Alvarez-Cermeño, Early differential diagnosis of multiple sclerosis using a new oligoclonal band test, Arch. Neurol. 62 (2005), pp. 574-577.

[29] W. Wang, P. Jones, and D. Partridge, Ranking pattern recognition features for neural networks, Proceedings of the First Conference on Advances in Pattern Recognition (ICAPR), Plymouth, England, 1998.

[30] D. Zeman, P. Adam, H. Kalistova, O. Sobek, J. Andel, and M. Andel, Cerebrospinal fluid cytologic findings in multiple sclerosis. A comparison between patient subgroups, Acta. Cytol. 45 (2001), pp. 51-59. 


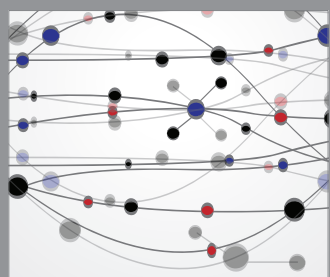

The Scientific World Journal
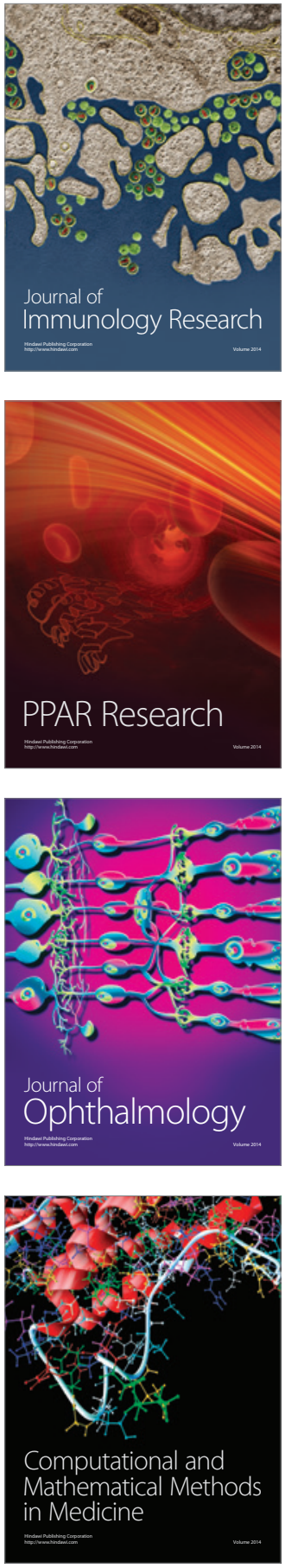

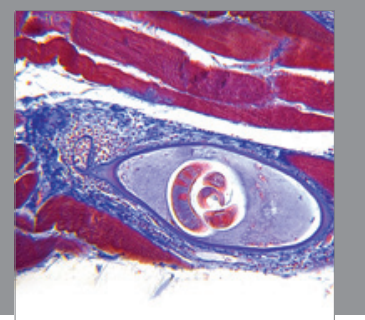

Gastroenterology

Research and Practice
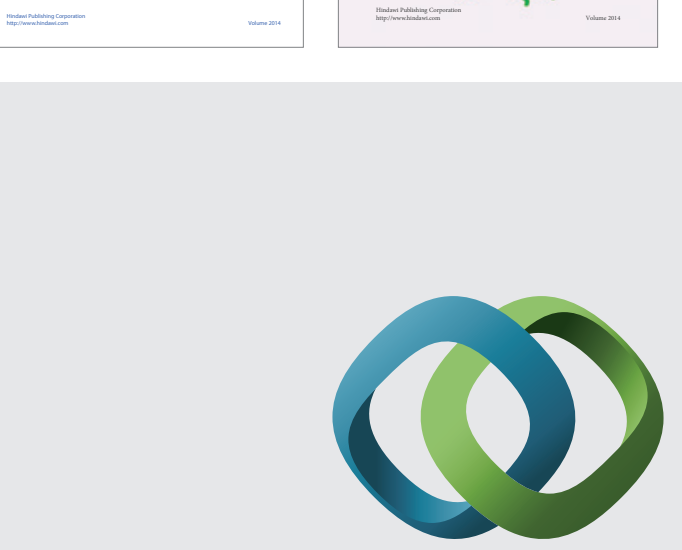

\section{Hindawi}

Submit your manuscripts at

http://www.hindawi.com
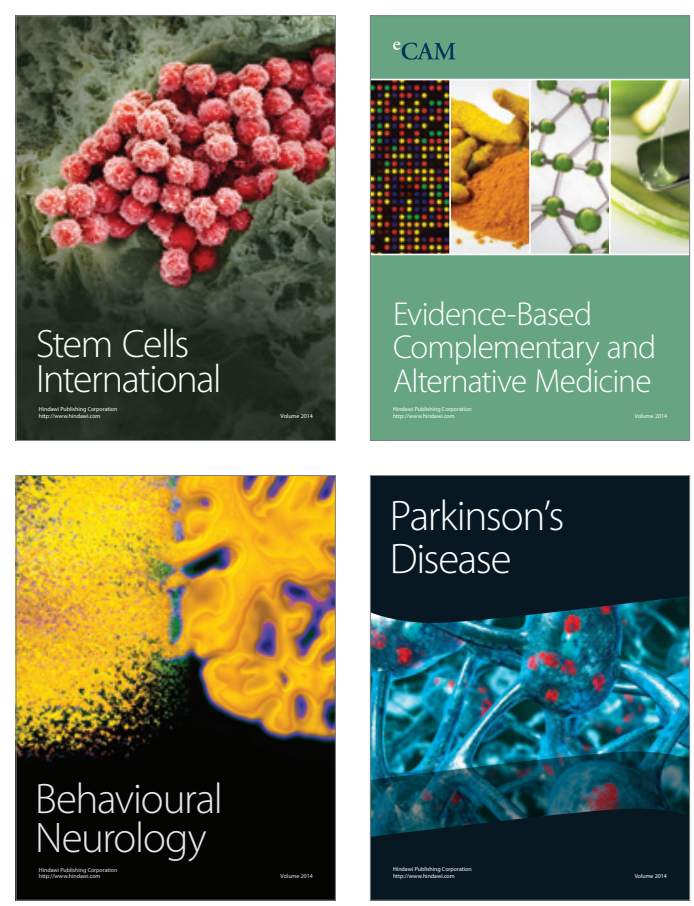

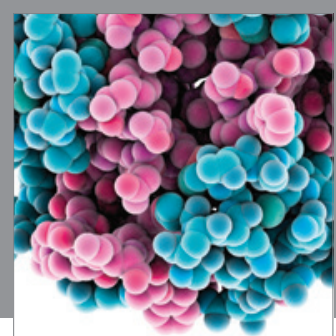

Journal of
Diabetes Research

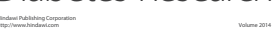

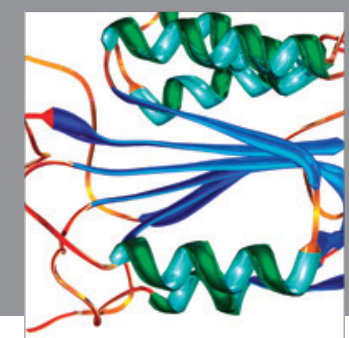

Disease Markers
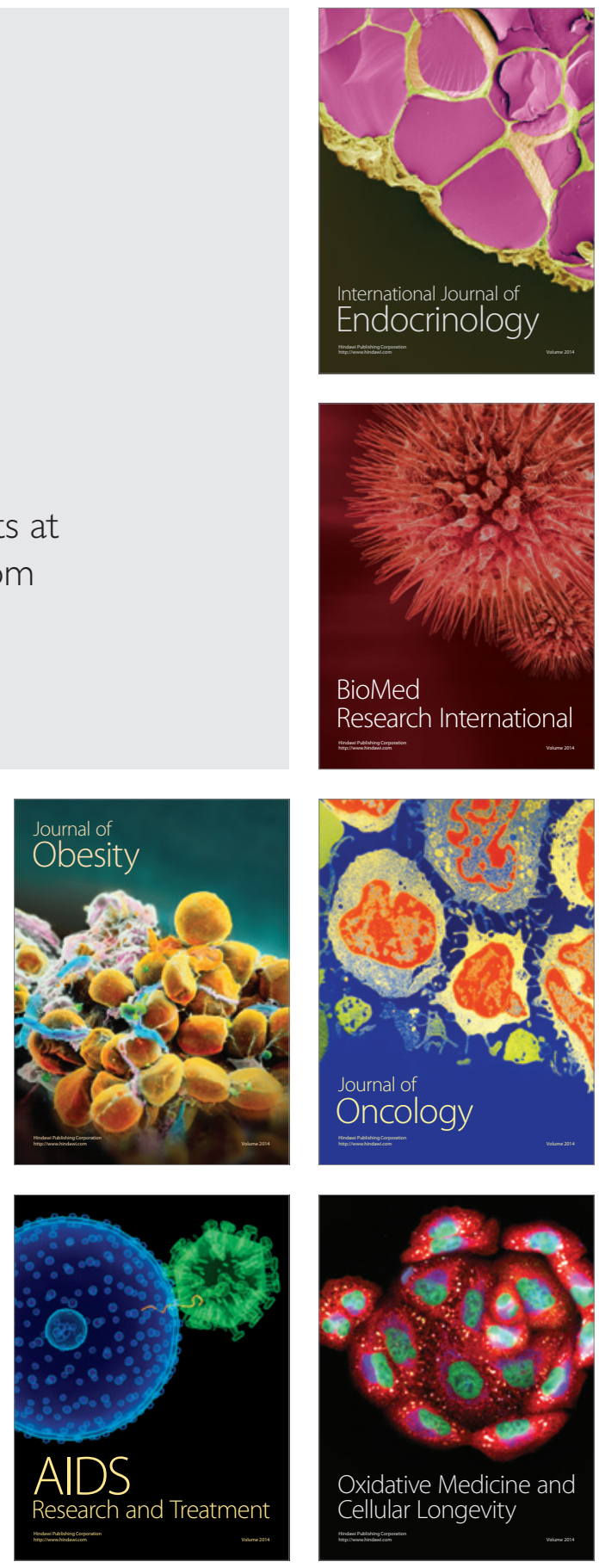